\title{
LATTICE LOCATION OF RARE EARTH IONS IN SEMICONDUCTORS AND THEIR OPTICAL ACTIVITY
}

\author{
A. Kozanecki \\ Institute of Pliysics, Polish Academy of Sciences \\ Al. Lotników 32/46, 02-668 Warszawa, Poland
}

\begin{abstract}
Lattice location experiments performed on Yb- and Er-doped III-V semiconducting compounds using Rutherford backscattering and channeling have been reviewed. It has been shown that $\mathrm{Yb}$ atoms locate substitutionally in InP and InP-based ternary alloys, while in gallium compounds no substitutional fraction of $\mathrm{Yb}$ could be detected. An intense intra- $f f$-shell luminescence of $\mathrm{Yb}^{3+}$ has been observed in In compounds. The photoluminescence spectra of $\mathrm{Yb}^{3+}$ reflect local alloy disorder in InPAs and GaInP, suggesting that the $\mathrm{Yb}$ atoms are tetrahedrally coordinated. No Yb-related emission could be observed in gallium compounds, except a weak $\mathrm{Yb}^{3+}$ photoluminescence in GaP. An evidence has been presented that $\mathrm{Er}$ atoms introduced into III-V compounds locate predominantly at interstitial positions. In GaAs they move into tetrahedral lattice sites as a result of thermal annealing at temperatures higher than $600^{\circ} \mathrm{C}$. The location of Er atoms at substitutional positions is accompanied with the disappearance of the intra- $f f$-shell luminescence of $\mathrm{Er}^{3+}$. The reasons of the observed correlation of luminescence properties and positions of $\mathrm{Er}$ and $\mathrm{Yb}$ atoms in zincblende lattices are discussed.
\end{abstract}

PACS numbers: 61.80.Ih, 78.55.-m

\section{Introduction}

Semiconducting materials doped with lanthanide ions have attracted much attention for their possible application in optoelectronic devices. These materials should combine the advantage of sharp, temperature stable, atomic-like electronic transitions within the $4 f$-shell of rare earth (RE) impurities with the ability of semiconductor to activate the emission by minority carrier injection. Sharp luminescence lines due to intra- $4 f$-shell transitions of REs have been observed in all the basic III-V materials [1-3] and in silicon [4].

Two lanthanide elements, $\mathrm{Er}$ and $\mathrm{Yb}$, are particularly interesting as impurities in semiconducting materials basic for microelectronics. Er-doped semiconductors have received considerable interest as prospective for applications in 
telecommunication, since the intra- $4 f$-shell luminescence of $\mathrm{Er}^{3+}$ occurring at a wavelength of $1.54 \mu \mathrm{m}$, corresponds to the minimum absorption of silica-based fibers. On the other hand, a simple $4 f^{13}$ electronic structure of the $\mathrm{Yb}^{3+}$ ion makes it ideal for studying the energy levels introduced by REs into forbidden gap of semiconductor, and the excitation and quenching mechanisms of intra-atomic luminescence by charge carriers.

In spite of intense technological effort to grow the high quality RE-doped semiconductors, reproducible optical and electrical properties of RE-related centres could be obtained only in Yb-doped InP and InP-based alloys [2, 5]. Poor reproducibility and difficulties met in growing most of the RE-doped III-V compounds are caused by two major reasons at least. One of them is due to the very high chemical activity of REs. On the other hand, structural misfit between the fourfold coordinated zincblende-type lattice of semiconductors and the sixfold coordination of the RE atoms typical of their stoichiometric compounds with As and $\mathrm{P}$ (pnictides) - a cubic $\mathrm{NaCl}$ structure [6] - may prevent the $\mathrm{RE}$ atoms to locate in regular tetrahedral positions.

Lattice constants of pnictides are very close to those of III-V semiconductors. However, as regards of the structural mismatch the bond lengths between host atoms in III-V compounds are at least $15 \%$ smaller than in pnictides, even if the lattice constants are equal. Moreover, the angles between bonds are different, and the RE-As (P) bonds in pnictides are much stronger than bonds between host atoms in semiconductors. It suggests that the RE-As or RE-P complexes, typical of pnictides, formed in crystalline lattice of semiconductor are resistant against thermal decomposition. The existence of such complex centres will lead to significant local lattice distortion, which may limit the efficiency of excitation of intra-atomic luminescence, i.e. by nonradiative Auger energy transfer from recombining charge carriers or excitons [7]. It also seems that RE atoms involved in such complexes will probably tend to locate nonsubstitutionally in the lattice. From these reasons the RE atoms incorporated into zincblende-type lattices of semiconductors can hardly be considered as isolated entities.

The problems mentioned above clearly points to the necessity of determining the location of RE ions in zincblende-type materials. This paper summarizes the experimental data concerning this subject for two RE-impurities commonly used in III-V compounds - Er and $\mathrm{Yb}$. Also, an interesting correlation between the location of REs and the possibility of exciting the intra- $4 f$-shell luminescence is presented.

\section{Rutherford backscattering and channeling in crystals}

Rutherford backscattering (RBS) and channeling spectroscopy seems to be the most suitable technique for determining the location of heavy ions in the lattice. The principle of the lattice location technique has been presented in Fig. 1. In the simplest case an RBS experiment has to be performed in two configurations. First of all an axial channeling spectrum is recorded. (Fig. 1a). In this measurement only interstitial atoms, apart from atoms at the surface, serve as scattering centres for incident analyzing ion beam. Next, the so-called random spectrum 


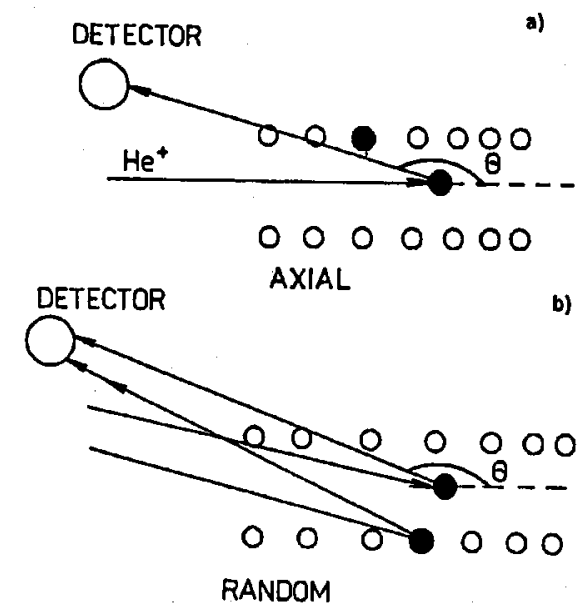

Fig. 1. The simplest way of determination the lattice location of impurity atom using RBS/channeling: (a) axial alignment, (b) random spectrum.

is collected (Fig. 1b), from which the total concentration of the impurity atoms can be calculated. The ratio of the channeling/random backscattering yields provides an approximate interstitial fraction of the impurity atoms. Determination of the exact position of an atom in the lattice requires angular dependence of the backscattering yield to be measured and model calculations has to be performed. (For detailed description see [8].)

\section{Lattice location of $\mathbf{Y b}$ impurity atoms in III-V compounds}

Most of lattice location experiments were performed on RE-implanted materials, since ion implantation is a well controllable technique, which allows one to get rid of many troubles caused by the high chemical activity of REs. A serious disadvantage of implantation is associated with extended damage produced by energetic ions, therefore a proper annealing procedure had to be applied to remove defects and activate impurities.

In Fig. 2 the profiles of $\mathrm{Yb}$ implanted into $\mathrm{InP}$ at a temperature of $250^{\circ} \mathrm{C}$ (to avoid amorphization of the surface layer) are presented. A comparison of the $\langle 100\rangle$ axial channeling and random spectra shows that at least $50 \%$ of the $\mathrm{Yb}$ atoms $\left(8 \times 10^{19} \mathrm{~cm}^{-3}\right)$ are located at substitutional positions [9]. In $\mathrm{Ga}_{0.5} \operatorname{In}_{0.5} \mathrm{P}$ implanted with $\mathrm{Yb}$ a smaller $\left(3 \times 10^{19} \mathrm{~cm}^{-3}\right)$ substitutional fraction of $\mathrm{Yb}$ atoms has also been found [9]. Similar measurements performed on GaP [10], GaAs, AlGaAs (Fig. 3) and GaAsP [9] showed that in all these materials the $\mathrm{Yb}$ atoms are located exlusively at nonsubstitutional positions.

In general, the Yb-related photoluminescence (PL) has not been observed in gallium compounds, except GaP in which a weak Yb PL could be detected [9]. Similarly, Takahei et al. [11] confirmed that $\mathrm{Yb}$ even in MOCVD grown GaAs:Yb 


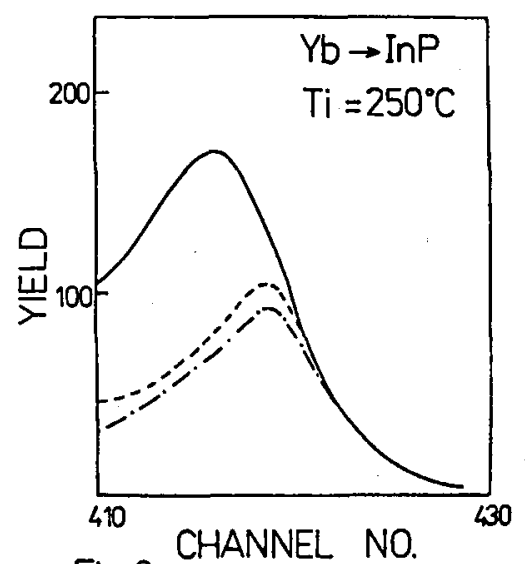

Fig. 2

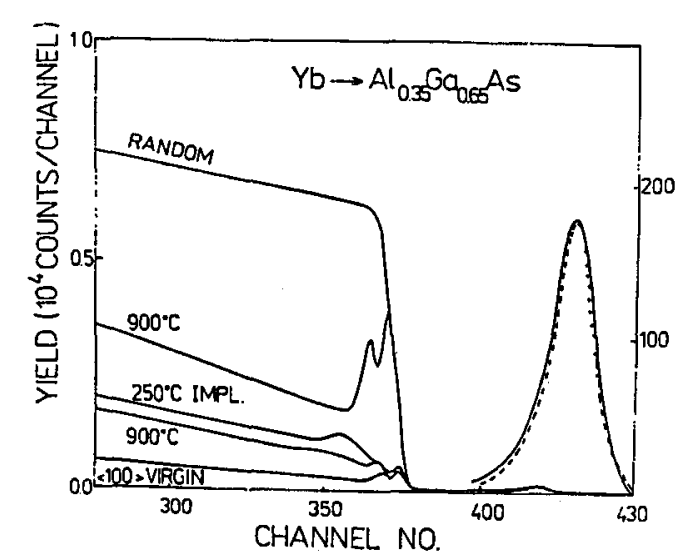

Fig. 3

Fig. 2. Random and $\langle 100\rangle$ axial profiles of $\mathrm{Yb}$ implanted into $\mathrm{InP}$ at $250^{\circ} \mathrm{C}$ [9]

Fig. 3. Random and $\langle 100\rangle$ axial profiles of $\mathrm{Yb}$ implanted into AlGaAs [9].

epilayers is inactive. They suggested that an electronic level associated with the $\mathrm{Yb}$ impurity is located close to the middle of the forbidden gap of GaAs, therefore the energy transferred from recombining charge carriers to the $4 f$-shell of $\mathrm{Yb}^{3+}$ is too low to excite the $4 f$-electron shell.

\section{Local alloy disorder probing with $\mathrm{Yb}^{3+}$ ions}

As it was mentioned above the efficient Yb-related PL was observed only in InP and in InP-based ternary alloys. In Fig. 4 the PL spectra of $\mathrm{Yb}^{3+}$ for selected compositions of GaInP implanted with $\mathrm{Yb}$ have been presented. It can be seen that the $\mathrm{Yb}$ spectra consist of two independent emissions which, in the authors opinion [12], are related to two different $\mathrm{Yb}$-centres: a cubic $\mathrm{Yb}_{-} \mathrm{P}_{4}$ and an axial one $\mathrm{Yb}-\mathrm{P}_{4}-\mathrm{In}_{11} \mathrm{Ga}$. Interestingly, the energy splitting $\left(\approx 9 \mathrm{~cm}^{-1}\right)$ of new PL lines labelled $B$ and $C$ is independent of alloy composition. It has been proposed [12] that the $B$ and $C$ lines are due to electron transition from the lowest lying level in the ${ }^{2} F_{5 / 2}$ excited state and the $\Gamma_{8}$ level in the ${ }^{2} F_{7 / 2}$ ground state of $\mathrm{Yb}^{3+}$ ion split to two components by uniaxial strain. The splitting is generated by the presence of a $\mathrm{Ga}$ atom in the position next-near-neighbour (NNN) to the $\mathrm{Yb}^{3+}$ ion. Following this interpretation it has been concluded that the local strain around the $\mathrm{Yb}$ atom is independent of alloy composition. In fact, the nearest environment of substitutional $\mathrm{Yb}^{3+}$ ions remains unchanged (4 P-atoms) in the full range of GaP-InP compositions. Moreover, the bond lengths between atoms change only slightly from InP to GaP [13].

In Fig. 5 the $\mathrm{Yb}$ PL spectra for InP, $\mathrm{Ga}_{0.5} \operatorname{In}_{0.5} \mathrm{P}$ and $\mathrm{GaP}$ have been presented. It can be seen that the spectrum for a $50 \%$ alloy follows the trend of Fig. 4 , and only for GaP the spectrum is shifted to higher energies. In our opinion the spectra in Fig. 5, particularly for $\mathrm{Ga}_{0.5} \operatorname{In}_{0.5} \mathrm{P}$, confirm that the local strain around 

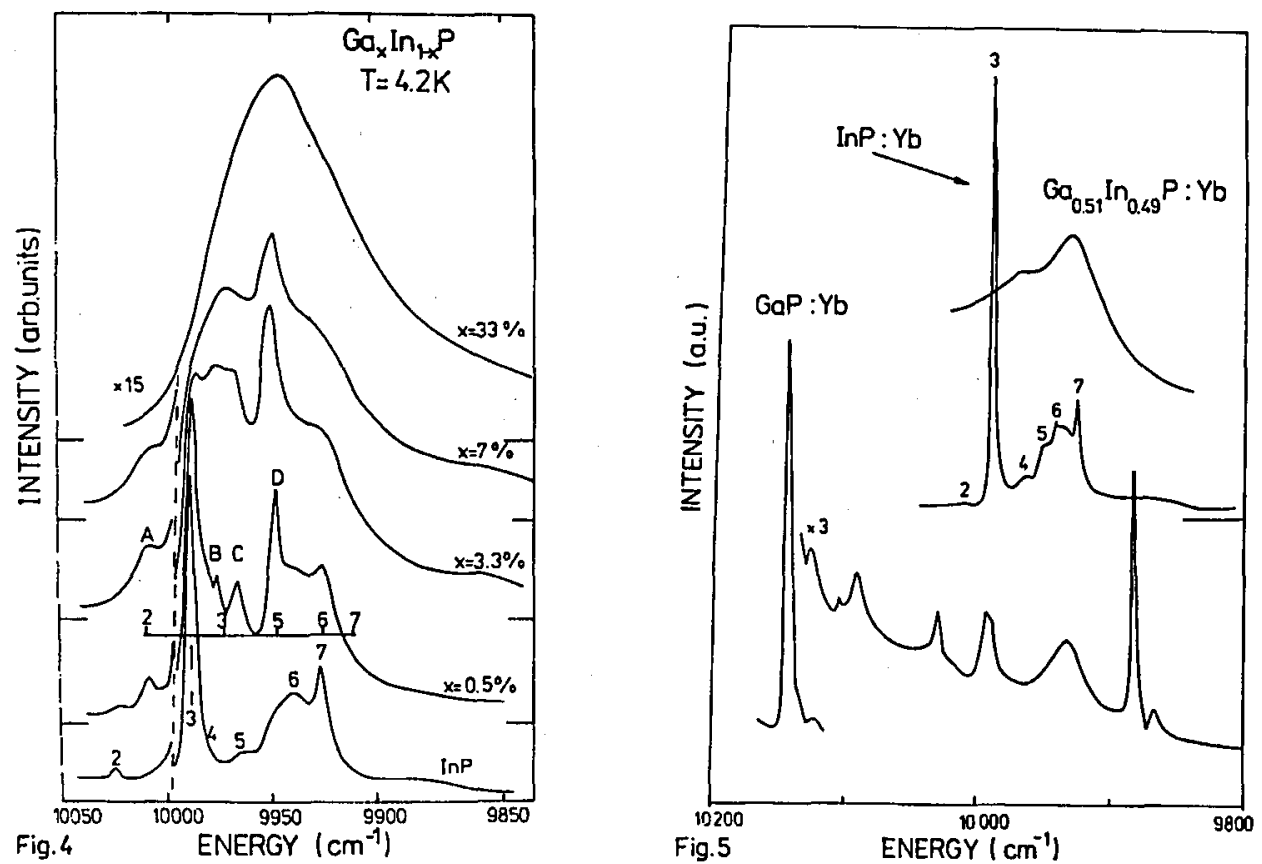

Fig. 4. $\mathrm{PL}$ spectra of $\mathrm{Yb}^{3+}$ in $\mathrm{Ga}_{x} \mathrm{In}_{1-x} \mathrm{P}$ alloys [12]

Fig. 5. A comparison of the $\mathrm{Yb} \mathrm{PL}$ spectra in $\operatorname{InP} \mathrm{Ga}_{0.5} \operatorname{In}_{0.5} \mathrm{P}$.

the $\mathrm{Yb}$ ion substituting an indium one is only weakly dependent on $\mathrm{Ga}$ contents.

A similar dependence on composition of $\mathrm{Yb}$ PL has been observed in InPAs alloys (Fig. 6) [14]. Once again the Yb-related spectra consist of two clearly resolved emissions. These two emissions have been assigned to two different $\mathrm{Yb}$-centrcs: a regular $\mathrm{Yb}-\mathrm{P}_{4}$ and an axial one $-\mathrm{Yb}-\mathrm{P}_{3} \mathrm{As}$ [14]. A small decrease in the transition energy of the emission ascribed to the axial centre seems to reflect an increase in the lattice constant when the As contents in InP increases.

Compositional dependence of Yb PL in alloys suggests that in spite of screening by the outer $5 s^{2}$ and $5 p^{6}$ closed shells, the $4 f$-electron wave function is affected by the presence of gallium and arsenic atoms in the mixed sublattices. As it is known isoelectronic impurities added to a binary compound change the interatomic distances in the common sublattice, while keeping the bond lengths almost preserved [13]. As a result the common sublattice suffers significant bond-angle distortions. Local distortions around impurities due to the shear component of the strain modify the intensity and symmetry of the electric field in the neighbourhood of the $\mathrm{Yb}$ atom. They may cause the Stark splittings of the $4 f$-electron energy levels of the $\mathrm{RE}^{3+}$-ion at a particular lattice site.

Interpretation of the Yb-related PL spectra in terms of random alloy splitting of electronic levels is supported by considering relaxation of host atoms in the nearest environment of $\mathrm{Yb}^{3+}$ ion. The bond lengths independent of alloy compo- 


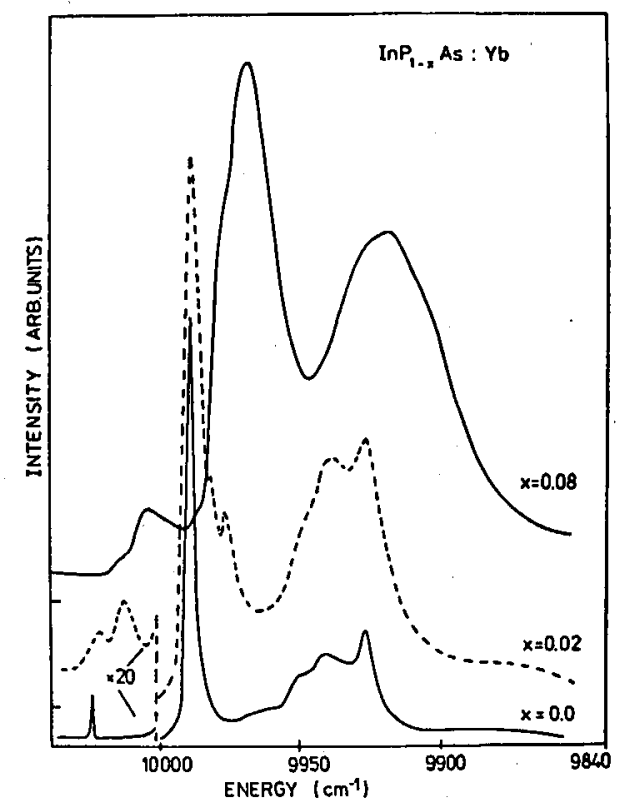

Fig. 6. PL spectra of $\mathrm{Yb}^{3+}$ for selected compositions of $\operatorname{InP}_{1-x} \mathrm{As}_{x}$ alloy.

sition cause large distortion of bonds of atoms which are bound to two or three atoms of different kind. It results in lowering the $T_{d}$ symmetry of the Yb-centre either to $C_{3 v}$ in case of $\mathrm{Yb}-\mathrm{P}_{3} \mathrm{As}$ complex or to $C_{s}$ in case of $\mathrm{Yb}-\mathrm{P}_{4}-\mathrm{In}_{11} \mathrm{Ga}$ centre. As a consequence, the $\Gamma_{8}$ quartets in the ${ }^{2} F_{7 / 2}$ ground and ${ }^{2} F_{5 / 2}$ excited states will split to two doublets giving rise to new PL transitions (Figs. 4 and 6 ).

In the light of channeling and optical measurements it can be concluded that the possibility of activation of $\mathrm{Yb}$ PL is connected to the location of $\mathrm{Yb}^{3+}$ ions in the lattice. Size matching of the $\mathrm{Yb}^{3+}$ and $\mathrm{In}^{3+}$ ions is one of the reasons that make the $\mathrm{Yb}-\mathrm{In}$ substitution possible. The partially covalent character of the $\mathrm{Yb}-\mathrm{P}$ bond [15] should also be favourable for substitutional location of $\mathrm{Yb}$. Moreover, covalent effects, understood as the admixture of ligand wave function to the $4 f$-electron wave function of $\mathrm{Yb}^{3+}$ ions relax the parity selection rule thus making parity forbidden dipole transitions in the $4 f$-shell possible [16].

\section{Erbium in III-V compounds}

A different kind of correlation between the possibility of exciting the intra- $4 f$ -shell luminescence and the location of RE atoms has been found for Er in III-V compounds. Kozanecki et al. [17] studied luminescence properties and lattice location of Er atoms implanted into GaAs at $250^{\circ} \mathrm{C}$. They have found that directly after implantation the $\mathrm{Er}$ atoms located predominantly at interstitial positions (Fig. 7). A weak Er-related luminescence could be observed after rapid thermal annealing within the $600-800^{\circ} \mathrm{C}$ temperature range. As a result of annealing the Er 


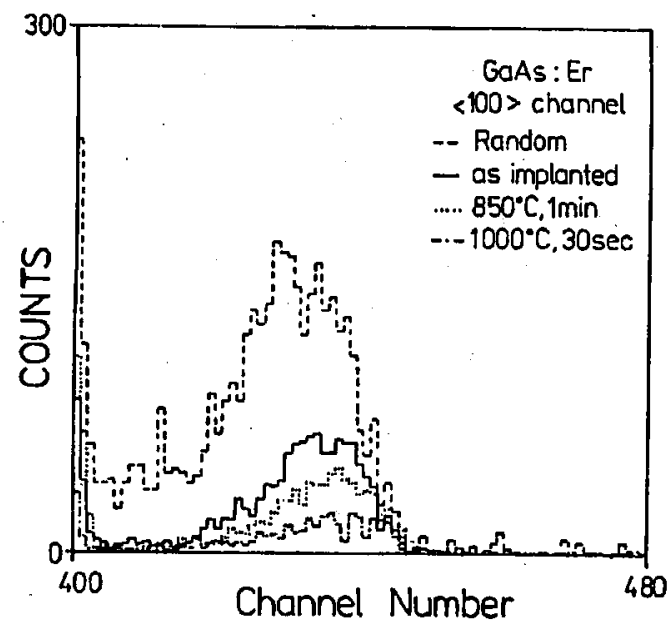

Fig. 7. Random and $\langle 100\rangle$ aligned profiles of Er atoms in as-implanted GaAs and thermally annealed GaAs [17].

atoms moved consecutively into substitutional positions and after $30 \mathrm{~s}$ at $1000^{\circ} \mathrm{C}$ they were virtually located in the $\langle 100\rangle$ atomic rows (Fig. 7). A fraction of $\mathrm{Er}$ atoms displaced from the $\langle 100\rangle$ row was at the level of few percent only. The $\langle 110\rangle$ aligned spectra have shown that the Er atoms were in fact displaced slightly from the substitutional lattice positions towards the $\langle 110\rangle$ channel [16]. Surprisingly, the intra- $4 f$-shell luminescence of $\mathrm{Er}^{3+}$ disappeared entirely when the majority of Er atoms had located substitutionally.

Observations on Er-implanted GaAs have been recently confirmed by lattice location measurements performed on molecular beam epitaxy (MBE) grown GaAs:Er epilayers [18]. Alves et al. [18] have shown that directly after the growth at $550^{\circ} \mathrm{C}$ the $\mathrm{Er}$ atoms were located at the tetrahedral interstitial positions. Intense intra-atomic luminescence of $\mathrm{Er}^{3+}$ could also be observed in as-grown samples. The authors have found that in MBE grown AlGaAs: $\mathrm{Er}$ a significant substitutional fraction of Er atoms exists, but no Er-related luminescence could be detected in this material [18].

In the light of channeling experiments performed on Er-implanted [17], MBE grown GaAs:Er, doping at the impurity level [18], and in ultra thin ErAs layers in GaAs [19], an interstitial location of optically active Er seems to be well established. However, the reasons of such unexpected correlation remains unclear. One of the possible reasons can be notorious affinity of Er to oxygen and the formation of $\mathrm{Er}_{2} \mathrm{O}_{3}$ precipitates in which $\mathrm{Er}^{3+}$ is optically active, as it was recently shown using EXAFS in Er-implanted silicon [20].

\section{References}

[1] V.A. Kasatkin, F.P. Kesamanly, B.E. Samorukov, Fiz. Tekh. Poluprovodn. 12, 1644 (1978). 
[2] H. Ennen, U. Kaufmann, G. Pomrenke, J. Schneider, J. Windscheiff, A. Axmann, J. Cryst. Growth 64, 165 (1984).

[3] K. Uwai, H. Nakagome, K. Takahei, Appl. Phys. Lett. 50, 977 (1987).

[4] Y.S. Tang, K.C. Heasman, W.P. Gillin, B.J. Sealy, Appl. Phys. Lett. 55, 432 (1989).

[5] K. Uwai, H. Nakagome, K. Takahei, Appl. Phys. Lett. 49, 1686 (1987).

[6] P. Villars, L.D. Calvert, Pearsons Handbook of Crystallographic Data for Intermetallic Phase, American Society of Metals, Metal Park, Ohio 1985.

[7] K. Takahei, A. Taguchi, H. Nakagome, K. Uwai, P.S. Whitney, J. Appl. Phys. 66, 4941 (1989).

[8] L.C. Feldman, J.W. Mayer, S.T. Picruax, Materials Analysis by Ion Channeling, Academic Press, New York 1982.

[9] A. Kozanecki, R. Groetzschel, J. Appl. Phys. 68, 517 (1990).

[10] A. Kozanecki, R. Groetzschel, J. Appl. Phys. 64, 3315 (1988).

[11] K. Takahei, A. Taguchi, in: Defects in Semiconductors 16, Eds. G. Davies, G. DeLeo, M. Stavola, Mater. Res. Forum, Vol. 83-87, Trans. Tech. Publ. Zürich 1991, p. 641.

[12] A. Kozanecki, Z. Kaliński, J. Raczyńska, J.M. Langer, J. Appl. Phys. 66, 3202 (1989).

[13] J.C. Mikkelsen Jr., J.B. Boyce, Phys. Rev. B 28, 7130 (1983).

[14] A. Kozanecki, Z. Kaliński, in: 20th Int. Conf. on the Physics of Semiconductors Thessaloniki (Greece) 1990, Eds. E.M. Anastassakis, J.D. Joannopoulos, World Scientific, Singapore 1990, p. 1989.

[15] G. Aszodi, J. Weber, Ch. Uhleihn, L. Pü-Lin, H. Ennen, U. Kaufmann, J. Schneider, J. Windscheiff, Phys. Rev. B 31, 7767 (1985).

[16] R. Boyn, Phys. Status Solidi B 148, 11 (1988).

[17] A. Kozanecki, M. Chan, C. Jeynes, B. Sealy, K. Homewood, Solid State Commun. 78, 763 (1991).

[18] E. Alves, M.F. da Silva, K.R. Evans, C.R. Jones, A.A. Melo, J. Soares, Nucl. Instrum. Methods Phys. Res. B 1993, in print.

[19] C.J. Palmström, N. Tabatabaie, S.J. Allen Jr., Appl. Phys. Lett. 53, 2608 (1988).

[20] D.L. Adler, D.C. Jacobson, D.J. Eaglesham, M.A. Marcus, J.L. Benton, J.M. Poate, P.H. Citrin, Appl. Phys. Lett. 61, 2181 (1992). 